\title{
Michal Šabo
}

On a maximal distance between graphs

Czechoslovak Mathematical Journal, Vol. 41 (1991), No. 2, 265-268

Persistent URL: http://dml.cz/dmlcz/102458

\section{Terms of use:}

(C) Institute of Mathematics AS CR, 1991

Institute of Mathematics of the Czech Academy of Sciences provides access to digitized documents strictly for personal use. Each copy of any part of this document must contain these Terms of use.

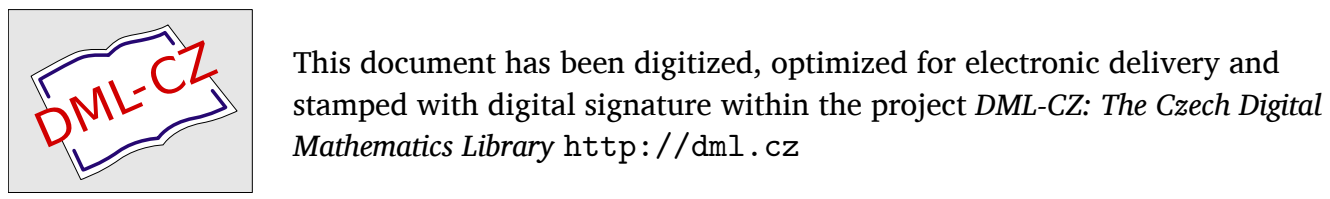




\title{
ON A MAXIMAL DISTANCE BETWEEN GRAPHS
}

\author{
Michal ŠABo, Bratislava
}

(Received January 25, 1989)

In [1], [2] some type of a metric for graphs was introduced. This type of a metric is based on the notion of maximal common subgraph (MCS). It is convenient e.g. for mathematical modelling of organic chemistry. This paper deals with the problem of a maximal distance between graphs in a given family of graphs. At the end of the paper, some problems of this theory are listed.

\section{PRELIMINARIES}

A graph $G=(V, E)$ consists of a non-empty finite vertex set $V$ and edge set $E$. The graphs considered here are undirected without loops and multiple edges. A subgraph $H$ of the graph $G$ is a graph obtained from $G$ by deleting some edges and vertices, $H \subseteq G$. Every edge $x \in E$ can be written by $x=(u, v)$, where $u, v \in V$ are vertices connected by the edge $x$. Two graphs $G_{1}=\left(V_{1}, E_{1}\right)$ and $G_{2}=\left(V_{2}, E_{2}\right)$ are isomorphic iff there exists $1-1$ correspondence $f: V_{1} \rightarrow V_{2}$ such that $(u, v) \in E_{1}$ if and only if $(f(u), f(v)) \in E_{2}, G_{1} \cong G_{2}$.

A graph $G$ is the common subgraph of the graphs $G_{1}, G_{2}$ iff there exist $H_{1}, H_{2}$ such that $H_{1} \subseteq G_{1}, H_{2} \subseteq G_{2}$ and $H_{1} \cong G, H_{2} \cong G$. A maximal common subgraph (MCS) is the common subgraph which contains the maximal number of edges.

The distance of the graphs $G_{1}=\left(V_{1}, E_{1}\right)$ and $G_{2}=\left(V_{2}, E_{2}\right)$ is defined by

$$
d\left(G_{1}, G_{2}\right)=\left|E_{1}\right|+\left|E_{2}\right|-2\left|E_{1,2}\right|+|| V_{1}|-| V_{2}||,
$$

where $\left|E_{1}\right|,\left|E_{2}\right|,\left|V_{1}\right|,\left|V_{2}\right|$ are cardinalities of the edge sets and vertex sets respectively and $\left|E_{1,2}\right|$ is the number of edges of MCS.

Let $\mathscr{F}_{p, q}$ be the family of all graphs with $p$ vertices and $q$ edges. It is clear that for $G_{1}, G_{2} \in \mathscr{F}_{p, q}$

$$
\grave{d}\left(G_{1}, G_{2}\right)=2 q-2\left|E_{1,2}\right| .
$$

If we identify the isomorphic graphs then $\mathscr{F}_{p, q}$ with the distance $d$ is a metric space.

Without loss of generality we can suppose that all graphs in $\mathscr{F}_{p, q}$ have the same vertex set $V$. 


\section{DIAMETER OF A FAMILY OF GRAPHS}

We define

$$
\operatorname{diam} \mathscr{F}_{p, q}=\max \left\{d(G, H) ; G, H \in \mathscr{F}_{p, q}\right\} .
$$

Evidently, $\operatorname{diam} \mathscr{F}_{p, 0}=\operatorname{diam} \mathscr{F}_{p, 1}=0$. We shall try to find out or to estimate $\operatorname{diam} \mathscr{F}_{p, q}$ for arbitrary $p, q$. We remark that

$$
0 \leqq q \leqq\left(\begin{array}{l}
p \\
2
\end{array}\right)
$$

Theorem 1. Let $G_{1}, G_{2} \in \mathscr{F}_{p, q}$, where $q \geqq 1$. Then

$$
d\left(G_{1}, G_{2}\right) \leqq 2 q-2 .
$$

Proof. MCS of the graphs $G_{1}$ and $G_{2}$ contains at least one edge.

The consequence of this theorem is: $\operatorname{diam} \mathscr{F}_{p, q} \leqq 2 q-2$.

Theorem 2. Let $q \geqq 1$. Then $\operatorname{diam} \mathscr{F}_{p, q}=2 q-2$ iff $q \leqq \frac{1}{2} p$.

Proof. Let $q \leqq \frac{1}{2} p$ and $V=\left\{v_{1}, v_{2}, \ldots, v_{p}\right\}$. We construct $G_{1}=\left(V, E_{1}\right), G_{2}=$ $=\left(V, E_{2}\right)$, where

$$
\begin{aligned}
& E_{1}=\left\{\left(v_{1}, v_{2}\right),\left(v_{1}, v_{3}\right), \ldots,\left(v_{1}, v_{q+1}\right)\right\}, \\
& E_{2}=\left\{\left(v_{1}, v_{2}\right),\left(v_{3}, v_{4}\right), \ldots,\left(v_{2 q-1} v_{2 q}\right)\right\} .
\end{aligned}
$$

MCS of of these graphs consist of one edge only. Therefore $d\left(G_{1}, G_{2}\right)=2 q-2$. Using Theorem 1 we have diam $\mathscr{F}_{p, q}=2 q-2$. Conversely, let diam $\mathscr{F}_{p, q}=2 q-2$ and let $q>\frac{1}{2} p$. Then for any $G_{1}, G_{2} \in \mathscr{F}_{p, q}$ we have $\sum \operatorname{deg} v_{i}=2 q>p$, where $\operatorname{deg} v_{i}$ is number of edges incident with the vertex $v_{i}$. It implies the existence of vertices $u, v$ such that $\operatorname{deg} u \geqq 2$ in $G_{1}$ and $\operatorname{deg} v \geqq 2$ in $G_{2}$. Then MCS of the graphs $G_{1}, G_{2}$ contains at least iwo edges. Therefore $d\left(G_{1}, G_{2}\right) \leqq 2 q-4$ for any $G_{1}, G_{2} \in \mathscr{F}_{p, q}$. It contradicts the assumption.

Theorem 3. Let $\frac{1}{2} p<q \leqq p-1$. Then $\operatorname{diam} \mathscr{F}_{p, q}=2 q-4$.

Proof. Theorem 2 implies that diam $\mathscr{F}_{p, q}<2 q-2$. We construct $G_{1}=\left(V, E_{1}\right)$, $G_{2}=\left(V, E_{2}\right)$ such that

$$
\begin{aligned}
& E_{1}=\left\{\left(v_{1}, v_{2}\right),\left(v_{1}, v_{3}\right), \ldots,\left(v_{1}, v_{q+1}\right)\right\}, \\
& E_{2}=\left\{\left(v_{1}, v_{2}\right),\left(v_{2}, v_{3}\right), \ldots,\left(v_{q}, v_{q+1}\right)\right\} .
\end{aligned}
$$

Then $G_{1}, G_{2} \in \mathscr{F}_{p, q}$ and their MCS contains two edges only. Therefore $d\left(G_{1}, G_{2}\right)=$ $=2 q-4$. It implies $2 q-4 \leqq \operatorname{diam} \mathscr{F}_{p, q}$. It proves that diam $\mathscr{F}_{p, q}=2 q-4$.

A complement of the graph $G=(V, E)$ is a giaph $\bar{G}=(V, \bar{E})$ which contains just the cdgcs which don't belong to $E$. It is clear that $|E|+|\bar{E}|=\frac{1}{2} p(p-1)$. In [3], it was proved:

Theorem 4. For arbitrary graphs $G, H$ with the same number of vertices the following holds: $d(G, H)=d(\bar{G}, \bar{H})$. 
Theorem 5. If we denote $\bar{q}=\frac{1}{2} p(p-1)-q$ then

$$
\operatorname{diam} \mathscr{F}_{p, q}=\operatorname{diam} \mathscr{F}_{p, \bar{q}}
$$

Theorem 6. Let $q \geqq \frac{1}{2} p(p-2)$. Then

$$
\operatorname{diam} \mathscr{F}_{p, q}=p(p-1)-2 q-2 .
$$

Proof. If $q \geqq \frac{1}{2} p(p-2)$ then

$$
\bar{q}=\frac{p}{2}(p-1)-q \leqq \frac{p}{2}(p-1)-\frac{p}{2}(p-2)=\frac{p}{2} .
$$

Using Theorem 2 and Theorem 5 we get

$$
\operatorname{diam} \mathscr{F}_{p, q}=\operatorname{diam} \mathscr{F}_{p, \bar{q}}=2 \bar{q}-2=p(p-1)-2 q-2 .
$$

Theorem 7. Let

$$
\left(\begin{array}{c}
p-1 \\
2
\end{array}\right) \leqq q<\frac{p}{2}(p-2)
$$

Then

$$
\operatorname{diam} \mathscr{F}_{p, q}=p(p-1)-2 q-4 .
$$

Proof. The inequality

$$
\left(\begin{array}{c}
p-1 \\
2
\end{array}\right) \leqq q<\frac{p}{2}(p-2)
$$

follows $\frac{1}{2} p<\bar{q} \leqq p-1$. Then

$$
\operatorname{diam} \mathscr{F}_{p, q}=\operatorname{diam} \mathscr{F}_{p, \bar{q}}=2 \bar{q}-4=p(p-1)-2 q-4 .
$$

\section{PROBLEMS}

It would be interesting to solve some problems connected with the notion of distance and diameter. We found out

$$
\operatorname{diam} \mathscr{F}_{p, q} \text { for } q \leqq p-1 \text { or } q \geqq\left(\begin{array}{c}
p-1 \\
2
\end{array}\right) \text {. }
$$

It implies that we know all diam $\mathscr{F}_{p, q}$ for $p \leqq 4$.

Problem 1. How to find out or estimate diam $\mathscr{F}_{p, q}$ for

$$
4<p \leqq q<\left(\begin{array}{c}
p-1 \\
2
\end{array}\right) ?
$$

The next problems are connected with the problems of distance between graphs with the sanme number of vertices and different number of edges.

Problem 2. If $G_{1} \in \mathscr{F}_{p, q}, G_{2} \in \mathscr{F}_{p, q_{2}}, q_{1}, q_{2} \geqq 1$ then

$$
d\left(G_{1}, G_{2}\right) \leqq q_{1}+q_{2}-2 .
$$

Under which conditions the equality holds? 
Problem 3. Obviously,

$$
\operatorname{diam}\left(\mathscr{F}_{p, q_{1}} \cup \mathscr{F}_{p, q_{2}}\right) \geqq \max \left(\operatorname{diam} \mathscr{F}_{p, q_{1}}, \operatorname{diam} \mathscr{F}_{p, q_{2}}\right) \text {. }
$$

For which $p, q_{1}, q_{2}$

a) $\operatorname{diam}\left(\mathscr{F}_{p, q_{1}} \cup \mathscr{F}_{p, q_{2}}\right)>\max \left(\operatorname{diam} \mathscr{F}_{p, q_{1}}\right.$, diam $\left.\mathscr{F}_{p, q_{2}}\right)$,

b) $\operatorname{diam}\left(\mathscr{F}_{p, q_{1}} \cup \mathscr{F}_{p, q_{2}}\right)=\max \left(\operatorname{diam} \mathscr{F}_{p, q_{1}}\right.$, diam $\left.\mathscr{F}_{p, q_{2}}\right)$ ?

Problem 4. Is any relation between $\operatorname{diam}\left(\mathscr{F}_{p, q_{1}} \cup \mathscr{F}_{p, q_{2}}\right)$ and $\operatorname{diam} \mathscr{F}_{p, q_{1}}+$ $+\operatorname{diam} \mathscr{F}_{p, q_{2}}$ ? Are there any non-trivial $p, q_{1}, q_{2}$ such that these numbers are the same?

Problem 5. Prove or reject the conjecture: If

$$
q_{1} \leqq q_{2} \leqq \frac{1}{2} \frac{p}{2}(p-1)
$$

then $\operatorname{diam} \mathscr{F}_{p, q_{1}} \leqq \operatorname{diam} \mathscr{F}_{p, q_{2}}$.

The last problem deals with the distance of graphs which have different numbers of vertices and edges. It is clear

$$
d\left(G_{1}, G_{2}\right) \leqq q_{1}+q_{2}+\left|p_{1}-p_{2}\right|-2
$$

for $G_{1} \in \mathscr{F}_{p_{1}, q_{1}}, G_{2} \in \mathscr{F}_{p_{2}, q_{2}}, q_{1}, q_{2} \geqq 1$.

Problem 6. Under which conditions

a) $d\left(G_{1}, G_{2}\right)=q_{1}+q_{2}+\left|p_{1}-p_{2}\right|-2$,

b) $d\left(G_{1}, G_{2}\right)=q_{1}+q_{2}+\left|p_{1}-p_{2}\right|-4$ hold?

Remark. B. Zelinka [4] solved the problem of diam $\mathscr{F}_{p}$, where $\mathscr{F}_{p}=\bigcup_{q} \mathscr{F}_{p, q}$ is the family of all graphs with $p$ vertices. He proved that diam $\mathscr{F}_{p}=\frac{1}{2} p(p-1)$.

\section{References}

[1] V. Baláž, J. Koča, V. Kvasnička, M. Sekanina: Metric for Graphs. Čas. Pest. Mat. 111 (1986), $431-433$.

[2] M. A. Johnson: Relating Metrics, Lines and Variables Defined on the Space of Graphs. Proceedings of the Fifth International Conference on Graph Theory. Eds. Y. Alavi, G. Chartrand, L. Lesniak and C. Wall. John Wiley. New York (1985), 457-470.

[3] V. Baláž, V. Kvasnička, J. Pospichal: Dual Approach for Edge Distance between Graphs. Čas. Pest. Mat. 114, No 2 (1989), 155-159.

[4] B. Zelinka: Edge Distance between Isomorphism Classes of Graphs. Čas. Pest. Mat. 112 (1987), 233.

Author's address: 81237 Bratislava, Radlinského 9, Czechoslovakia (Katedra matematiky CHTF SVŠT). 